\title{
Vernarbende Alopezie bei einer 27-jährigen Frau
}

\section{Cicatricial Alopecia in a 27-Year-Old Woman}

Autoren

Institut
D. Helbig', J. C. Simon ${ }^{1}$, U. Paasch ${ }^{1}$

Klinik und Poliklinik für Dermatologie, Venerologie und Allergologie der Universität Leipzig

\section{Bibliografie}

DOI http://dx.doi.org/

10.1055/s-0029-1243889

Online-Publikation: 4. 2. 2010

Akt Dermatol 2010; 36:

226-228 ๑ Georg Thieme

Verlag KG Stuttgart · New York ISSN 0340-2541

\section{Korrespondenzadresse}

Dr. med. Doris Helbig

Klinik und Poliklinik für

Dermatologie, Venerologie

und Allergologie

Universität Leipzig

Philipp Rosenthal Str. 23

04103 Leipzig

doris.helbig@medizin.

uni-leipzig.de

\section{Zusammenfassung \\ $\nabla$}

Eine 27-jährige Patientin stellte sich mit einer seit 2 Jahren bestehenden, progredienten, therapierefraktären Alopezie vor. Im Bereich des zentralen, parietookzipitalen Kapillitiums zeigte sich ein ca. $12 \times 10 \mathrm{~cm}$ großes alopezisches, vernarbendes Areal. Histologisch zeigten sich in den oberen Dermisabschnitten mehrere Haarschäfte innerhalb dilatierter Follikel mit umgebenden gemischtzelligen Entzündungszellinfiltraten und Fibrose. Die mikrobiologischen Untersuchungen führten zum Nachweis von Staphylococcus aureus. Aufgrund des Nachweises von Staphylococ-

\section{Einleitung \\ $\nabla$}

Die Büschelhaarfollikulitis bzw. „tufted hair folliculitis“, erstbeschrieben von Smith und Sanderson 1978 [1], ist eine seltene Erkrankung, welche durch das Austreten multipler Haarschäfte aus dilatierten Follikelöffnungen charakterisiert wird. Die von einigen Autoren als Variante der Folliculitis decalvans aufgefasste Follikulitis tritt vor allem in parietalen und okzipitalen Regionen der Kopfhaut auf. Ihre Pathogenese ist nicht vollständig geklärt, oftmals können jedoch bakterielle Infektionen nachgewiesen werden. Wir berichten über eine 27-jährige Patientin mit Büschelhaarfollikulitis, geben einen Überblick über pathogenetische Hypothesen und Therapieoptionen.

\section{Kasuistik \\ $\nabla$}

\section{Anamnese}

Eine 27-jährige Patientin stellte sich mit einer seit 2 Jahren bestehenden, progredienten Alopezie vor. Lokaltherapien mit verschiedenen lokalen Glukokortikoiden hatten zu keiner Besserung geführt. cus aureus im Abstrich leiteten wir eine systemische antibiotische Therapie ein, deren Wirkung abzuwarten bleibt. Die Büschelhaarfollikulitis bzw. "tufted hair folliculitis“ ist eine seltene, pathogenetisch nicht vollständig verstandene Erkrankung, welche durch das Austreten multipler Haarschäfte aus dilatierten Follikelöffnungen charakterisiert wird. Obwohl nicht immer bakterielle Erreger detektiert werden können, scheinen bakterielle Infektionen eine entscheidende Rolle in der Entstehung der Büschelhaarfollikulitis zu spielen, sodass sich der Versuch einer systemischen antibiotischen Therapie bei wenigen Therapiealternativen anbietet.

\section{Erst-|Aufnahmebefund}

Im Bereich des zentralen, parietookzipitalen Kapillitiums zeigte sich ein ca. $12 \times 10 \mathrm{~cm}$ großes haarloses Areal ohne nachweisbare Follikel. Im Randbereich wurden vereinzelte abgebrochene Haare und seröse Krusten sichtbar $(\boldsymbol{A}$ Abb. 1 und 2). Der weitere Untersuchungsbefund inklusive der Palpation der Lymphknoten war unauffällig.

\section{Befunde diagnostischer Untersuchungen}

Histologisch zeigte sich eine ortholog strukturierte Epidermis mit abgeflachten Reteleisten und scharfer Abgrenzung zum darunter liegenden Bindegewebe. In den oberen Dermisabschnitten konnten innerhalb eines dilatierten Follikelganges mehrere Anschnitte von Haarschäften nachgewiesen werden. Die Haarbulbi in der tieferen Dermis- bzw. Subkutis stellten sich regelhaft dar. Perivaskulär und perifollikulär formierten sich Entzündungszellinfiltrate, bestehend aus Lymphozyten, neutrophilen Granulozyten, Epitheloidzellen und Fremdkörper-Riesenzellen. Das interfollikuläre Bindegewebe war fibrosiert bei Reduktion der Haarfollikelzahl ( $\bullet$ Abb. 3 und 4). In der PAS-Färbung ergab sich kein Nachweis von 


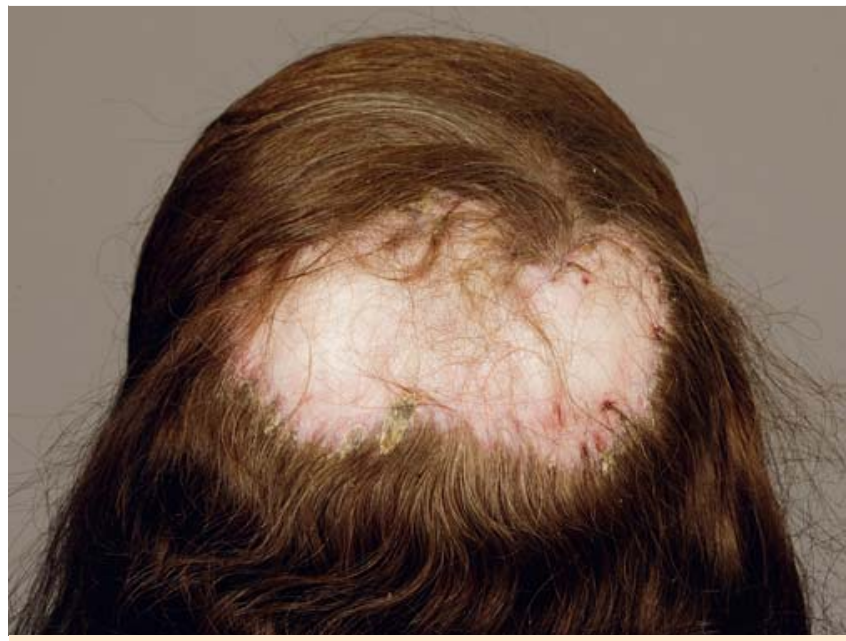

Abb. 1 Ca. $12 \times 10 \mathrm{~cm}$ großes haarloses Areal ohne nachweisbare Follikel und vereinzelten abgebrochenen Haaren und serösen Krusten im Randbereich.

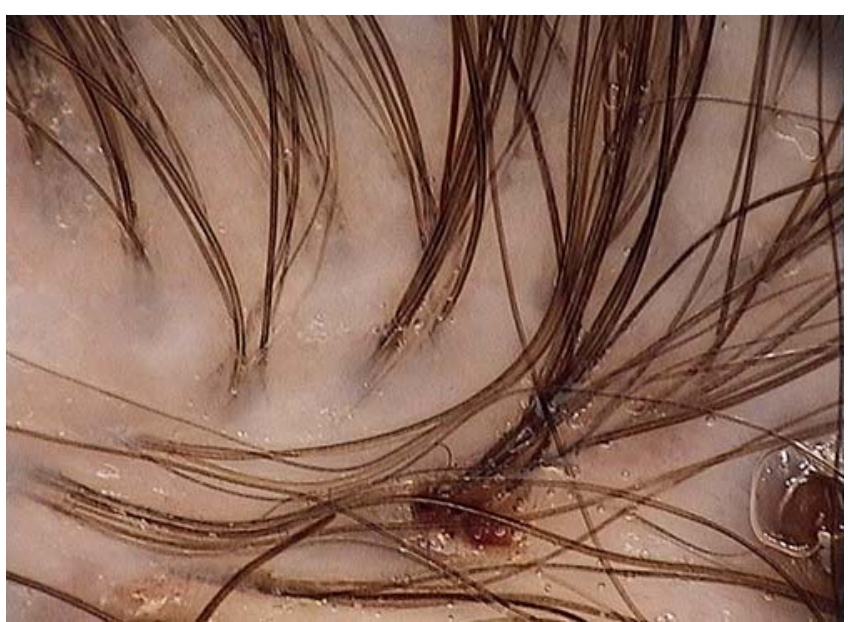

Abb. 2 Trichoscan: Haare treten gebündelt aus.

Pilzelementen. Die mikrobiologischen Untersuchungen führten zum Nachweis von Staphylococcus aureus.

Therapie und Verlauf

Aufgrund der histologischen Veränderungen in Kombination mit dem Nachweis von Staphylococcus aureus im Abstrich stellten wir die Diagnose einer Büschelhaarfollikulitis bzw. „tufted hair folliculitis“. Wir leiteten bei unserer Patientin eine Therapie mit Cefuroxim $2 \times 500 \mathrm{mg} / \mathrm{d}$ p.o. ein. Lokal wurde zusätzlich desinfizierend mit Octenisept ${ }^{\circledR}$ behandelt. Der weitere Verlauf bleibt abzuwarten.

\section{Diskussion}

$\nabla$

Die Büschelhaarfollikulitis bzw. „tufted hair folliculitis“, erstbeschrieben von Smith und Sanderson 1978 [1], ist eine seltene Erkrankung, welche durch das Austreten multipler Haarschäfte aus einer dilatierten Follikelöffnung charakterisiert wird. Von einigen Autoren als Variante der Folliculitis decalvans aufgefasst, zeigen sich klinisch perifollikuläre Erytheme und follikuläre Pusteln

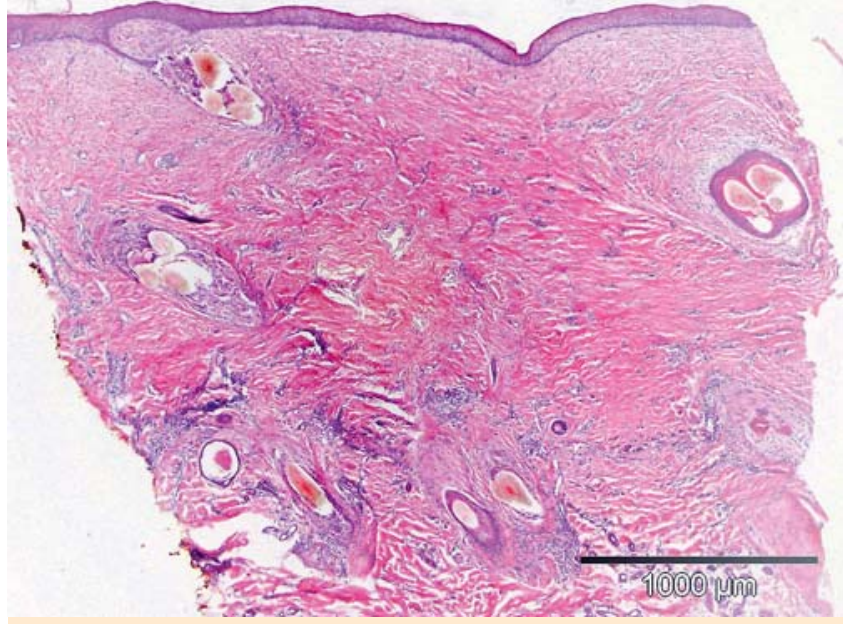

Abb. 3 (Histologie Übersicht; $4 \times \mathrm{HE}$ ): Ortholog strukturierte Epidermis mit abgeflachten Reteleisten. In der Dermis perivaskulär und perifollikulär akzentuierte, gemischtzellige Entzündungszellinfiltrate, Rarefizierung der Haarfollikel und interfollikuläre Fibrose.

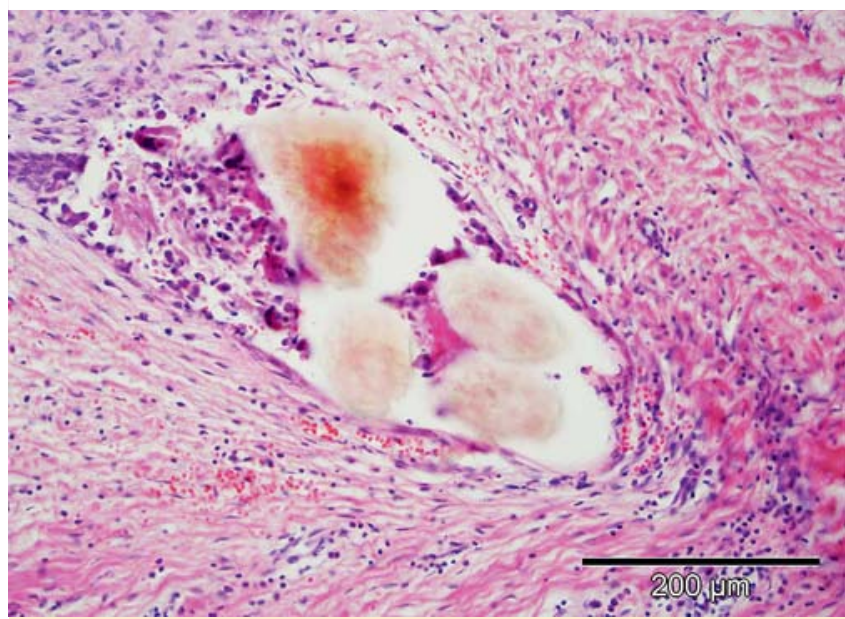

Abb. 4 (Histologie Detail; $20 \times \mathrm{HE}$ ): In den oberen Dermisabschnitten erkennt man innerhalb eines erweiterten Follikelganges mehrere Anschnitte von Haarschäften. Perifollikulär formiert sich ein Entzündungszellinfiltrat bestehend aus Lymphozyten, neutrophilen Granulozyten, Epitheloidzellen und Fremdkörper-Riesenzellen.

mit zentrifugaler Ausbreitungstendenz und resultierender vernarbender Alopezie. Betroffen sind vor allem parietale und okzipitale Regionen der Kopfhaut. Die Anagen-Telogen-Ratio, aber auch die Haarfollikeldichte kann bei der Büschelhaarfollikulitis einem Normalzustand entsprechen. Männer sind häufiger betroffen als Frauen, das Erkrankungsalter ist stark variabel.

Die Pathogenese der Büschelhaarfollikulitis ist nicht vollständig verstanden. Konfluierende Nekrosen der oberflächigen Anteile mehrerer Haarfollikel bei nicht betroffenen Haarbulbi und persistierendem Haarwachstum führen zu dem Austreten mehrerer Haare durch einen Haarfollikelgang. Sekundär kann es zu zystischen Aufweitungen oder auch Rupturen der Haarfollikel mit folgender Fremdkörperreaktion kommen. Obwohl nicht immer bakterielle Erreger detektiert werden können und systemische antibiotische Therapien allein oft nicht ausreichen, scheinen Infektionen mit Staphylococcus aureus im Besonderen, aber auch Mischinfektionen mit Klebsiella oxytoca und Citrobacter species 
eine entscheidende Rolle in der Entstehung der Büschelhaarfollikulitis zu spielen. Störungen der zellulären Immunität und eine Hypokomplementämie stellen zudem prädestinierende Faktoren für follikuläre Infektionen dar. Lokale Traumata und diverse entzündliche Dermatosen des Kapillitiums inklusive des Pemphigus vulgaris [2], anderer vernarbender Alopezien wie der Folliculitis decalvans, Folliculitis keloidalis bzw. Keratosis follicularis spinulosa decalvans stellen weitere zugrundeliegende Erkrankungen dar [2]. Des Weiteren wurde das Auftreten von Büschelhaaren unter einer Therapie mit Lapatinib, einem oralen Tyrosinkinaseinhibitor zur Therapie fortgeschrittener Stadien HER2-positiver Mamma-Karzinome, beschrieben. Unter dieser Therapie kommt es durch die Blockade von EGF-Rezeptoren zu einem vermehrten follikulären Wachstum mit sekundärer Retention der Haare in Büscheln [3]. Abzugrenzen davon sind primäre Büschelhaare sowie Formen bei nävoiden Malformationen.

Therapeutisch kommen diverse systemische, breit wirksame Antibiotika inklusive Rifampicin und Clindamycin zum Einsatz [4]. Therapien mit Prednisolon, Isotretinoin und Methotrexat zeigten nur geringe Wirksamkeit.

Rezidive und progrediente Verläufe sind häufig und könnten Folge einer inadäquaten Immunantwort auf Staphylococcus aureus darstellen [4]. Im ausgebrannten Stadium kann die Exzision vernarbter Hautareale erwogen werden.

Interessenkonflikt: Die Autoren geben an, dass kein Interessenkonflikt vorliegt.

\section{Abstract}

\section{Cicatricial Alopecia in a 27-Year-Old Woman $\nabla$}

A 27-year-old woman presented with a progressive alopecia, persisting since two years. Examination revealed an area of cicatricial alopecia parieto-occipital, $12 \times 10 \mathrm{~cm}$ in diameter. Histopathological examination revealed multiple hair tufts emerging from dilated follicle openings surrounded by a mixed inflammatory infiltrate and fibrosis. Results of bacteriologic examination of pus extruding from the dilated follicular ostia revealed Staphylococcus aureus. The patient was treated with a systemic antibiotic therapy, whose effects still have to be awaited. Tufted hair folliculitis is a rare folliculitis with irregular areas of scarring alopecia within which numerous hair tufts emerge from dilated openings. Its pathogenesis is not fully understood. Bacterial infections seem to play an important causative role although bacterial infections cannot always be detected. For this reason, antibiotics administered systemically are a promising treatment option.

\section{Literatur}

1 Smith NP, Sanderson KV. Tufted folliculitis of the scalp. J R Soc Med 1978; 71: 606-608

2 Jappe $U$, Schroder K, Zillikens D, Petzoldt D. Tufted hair folliculitis associated with pemphigus vulgaris. J Eur Acad Dermatol Venereol 2003; 17: $223-226$

3 Ena P, Fadda GM, Ena L, Farris A, Santeufemia DA. Tufted hair folliculitis in a woman treated with lapatinib for breast cancer. Clin Exp Dermatol 2008; 33: 790-791

4 Powell JJ, Dawber RP, Gatter K. Folliculitis decalvans including tufted folliculitis: clinical, histological and therapeutic findings. Br J Dermatol 1999; 140 : $328-333$ 